\title{
La educación privada y el área científica favorecen la lectura de universitarios
}

Víctor Hugo Robles-Francia*

Adriana Mariela de la Cruz-Caballero**

Aída Dinorah García Álvarez*

Lenin Martínez Pérez*

Jorge Rebollo Meza*

Artículo recibido:

5 de junio de 2018

Artículo aceptado:

22 de octubre de 2018

Artículo de investigación

\section{Resumen}

Se reporta una investigación sobre la incidencia del género, del tipo de institución, pública o privada y del área científica en la dedicación a la lectura en formato impreso, en digital y en el uso de internet de 5455 estudiantes universitarios. Se realizó un análisis correlacional y univariado para la comprobación de las hipótesis, encontrando que el uso de internet se relaciona positivamente con la dedicación a la lectura en texto impreso y con la lectura en formato

\footnotetext{
* División Académica de Ciencias Económico Administrativas. Universidad Juárez Autónoma de Tabasco, México vicrob13@yahoo.com.mx adgaral@gmail.com leninmartinez@outlook.com jorge_rebollo_58@hotmail.com ** Instituto Tecnológico Superior de Centla, México adriana18100@ @otmail.com INVESTIGACIÓN BIBLIOTECOLÓGICA, vol.33, núm.78, enero/marzo, 2019, México,
} ISSN: 2448-8321 pp. 63-79 
digital. Se encontró, además, que la disciplina científica incide en la dedicación a la lectura impresa: los universitarios de Ciencias Sociales y Humanidades de universidades públicas manifestaron el mayor número de horas de dedicación. En la lectura digital, el género, el tipo de disciplina científica y de institución incidieron en las horas de dedicación: los universitarios de sexo masculino de Ciencias Básicas e Ingeniería de universidades privadas declararon el mayor número de horas de dedicación a la lectura digital.

Palabras clave: Lectura digital; TIC; Género; Disciplina Científica

Private Education and the Scientific Area, favor the Reading of University Students

Victor Hugo Robles-Francia, Adriana Mariela de la Cruz-Caballero, Aída Dinorah García-Álvarez, Lenin Martinez-Pérez and Jorge Rebollo-Meza

\section{Abstract}

This research reports about the incidence of gender, type of institution, public or private, and the scientific area, as regards the student's dedication to reading. Therefore 5455 university students were surveyed about: the Internet use, and the digital or printed reading. An univariate and a correlational analysis were carried out for verifying the two hypothesis and the results find that the use of internet is positively correlated with the students reading dedication to lecture, in both printed and digital formats. The Humanities and the Social Sciences students from public universities presented the greatest number of hours dedicated to lecture. As to digital reading: gender, type of scientific discipline and of institution, these trends affected the number of hours dedicated to lecture. Male university students of Basic Sciences and Engineering and private universities, showed the greatest digital reading dedication. Results, discussion and conclusions are included.

Keywords: Digital Reading; TIC; Gender; Scientific Discipline 


\section{INTRODUCCIÓN}

$\mathrm{C}^{1}$ apoyo a la lectura y a las bibliotecas es una de las principales metas Eeducativas de calidad para el alumnado y una pauta establecida por la Organización de Estados Iberoamericanos (OEI), que en frases de Marchesi (2001) busca apoyar estudios sobre lectura y formas de funcionamiento innovador de bibliotecas escolares. La presente indagatoria se enfocó en observar el uso de internet y la lectura en formato impreso y digital del alumnado de universidades, tanto públicas como privadas de las diversas disciplinas científicas, con el fin de describir las prácticas lectoras de los universitarios y, con ello, identificar innovaciones potenciales para las bibliotecas universitarias.

En la sociedad contemporánea, afirma Ramírez (2016), se requieren lectores idóneos capaces de crear conocimiento. Amiama-Espaillat y Mayor-Ruiz (2017) consideran a la lectura como una competencia multidimensional, sociocultural y vivencial, que integra gran variedad de saberes y comportamientos para integrar formatos, datos y modos de lectura diversos.

El desarrollo tecnológico y el internet brindan a los estudiantes más herramientas para desarrollar sus actividades, así como nuevas estrategias de lectura (Moya y Gerber, 2016). El alumnado debe desarrollar competencias informáticas, interacciones mediante dispositivos electrónicos donde lee frecuentemente, pero el acercamiento digital no implica el dominio de prácticas lectoras requeridas en el contexto académico (López, 2016). Ramírez (2016) afirma que debe promoverse el deseo por el mismo placer. Álvarez et al. (2009) secundan esta idea hedonista de alto valor simbólico y la de no separar la lectura de la alfabetización ni de la dimensión cultural, sino el goce social, la sensación placentera de construir lazos en una comunidad, con sentido individual de la lectura.

Amiama-Espaillat y Mayor-Ruiz (2017) afirman que la lectura es la base para una vida plena y activa en todas las áreas de la sociedad contemporánea, sin competencia lectora un ser humano no puede ser libre ni desarrollarse plenamente. Así, un lector contribuye a su desarrollo cognitivo, emocional, lúdico y social, adquiere una buena competencia comunicativa y es capaz de interactuar con textos tradicionales y digitales; por otra parte, se encuentra el individuo considerado analfabeto digital, que prefiere la información en formato visual y texto corto, suele comunicarse e informarse, pero no construye significado.

La lectura digital es la combinación de textos y recursos multimedia que se encuentran en un medio electrónico. Los textos digitales modifican la forma de lectura y de pensamiento (Amiama-Espaillat y Mayor-Ruiz, 2017). No obstante, Moya y Gerber (2016) reconocen en la lectura de formatos digitales 
una alarmante continuidad en las desigualdades sociales que han caracterizado a la lectura del formato impreso.

Respecto a la lectura en universidades públicas y privadas, la correspondencia entre posiciones sociales y prácticas culturales observa también una correlación. La lectura en formatos digitales se asocia con una menor edad en comparación con los impresos, la edad media de los grupos de lectores en ambos formatos o digital está debajo de los 34 años (Moya y Gerber, 2016).

Las diferencias lectoras y los inconvenientes en el acceso en la educación superior suelen ser marcados. Shandana y Hafsa (2015) descubrieron que existen obstáculos en el uso de las TIC entre los estudiantes de universidades públicas y privadas. Su estudio reveló que los estudiantes universitarios varones enfrentan más obstáculos en el uso de las TIC que las mujeres.

Arias (2015) muestra diferencias significativas en el uso de las TIC entre universidades públicas y privadas. Complementariamente, Amiama-Espaillat y Mayor-Ruiz (2017) encuentran que $84 \%$ de los estudiantes del sector público carece de la competencia lectora mínima, mientras que en el sector privado es $25 \%$. Los estudiantes del sector privado se ubican con niveles más altos de competencia lectora, el estudiante que ya posee los conocimientos previos y que vive en una cultura letrada incorpora de manera más eficiente lo leído, pero el que posee limitados conocimientos previos, por no estar inmerso en una cultura lectora, aunque lea mucho y utilice las TIC no podrá incorporar eficientemente la información.

Curbelo y Moreira (2014) exponen que se entiende por género a las formas históricas y socioculturales en que varones y mujeres interactúan y dividen sus funciones, donde el ser humano es socializado para realizar unas tareas u otras, existiendo sanciones sociales para quienes se desvíen del comportamiento esperado (Curbelo y Moreira, 2014). Los hombres poseen mayores destrezas en lo que refiere al uso de las TIC, perciben de buena a excelente su habilidad para usar computadoras. Pero Torres y Arras (2011) no encontraron diferencias significativas entre hombres y mujeres sobre las competencias TIC.

Moya y Gerber (2016) aseguran, en el caso de la lectura digital, que los lectores digitales, si bien tienen niveles de ingreso y educación similares, tienden a ser en mayor medida hombres $(60.3 \%)$ y a usar internet por un número superior de horas promedio a la semana que el resto de los grupos (10.27 horas). Según los resultados de estos autores, las mujeres prefieren un poco más la lectura impresa que los hombres y $50 \%$ de ambos géneros prefieren los dos formatos.

La lectura en formato impreso y digital suele tener diversas prácticas por los estudiantes de ciencias sociales, naturales, económicas o humanidades. En las ciencias sociales y humanidades (CSH), Salvador Oliván y Agustín Lacruz 
(2015) investigaron 561 estudiantes universitarios de humanidades en España, donde los estudiantes manifestaron ser lectores frecuentes de libros, mayormente impresos. Asimismo, un segmento importante de estos estudiantes leía material digital. Estos autores señalan que los estudiantes de humanidades de España prefieren el formato impreso, casi $80 \%$ contra $17 \%$ digital. Esto contradice lo encontrado por García y Arias (2014), que estudiantes españoles del área de sociales y humanidades, de periodismo, prefieren la lectura en formato digital.

En las ciencias básicas e ingeniería (CBI), Ramírez (2006) investigó a 325 alumnos de licenciatura en ciencias, física, química y matemáticas, entre otras, y a 353 de humanidades, filosofía, literatura, entre otras, de la Universidad Nacional Autónoma de México; encontró que $83.35 \%$ de los estudiantes empleaba en promedio una computadora cuatro horas al día, el porcentaje más alto $(76.48 \%$ ) se dio en las y los estudiantes en humanidades, que pasan entre una y tres horas al día. Asimismo, señala que $17.99 \%$ de los estudiantes de humanidades y ciencias prefirieron leer en la pantalla en lugar del formato en texto impreso, no hubo diferencias significativas entre los estudiantes de humanidades y de ciencias ni entre hombre y mujeres.

En estudiantes de ingeniería, Cornejo et al. (2012) encontraron que 158 estudiantes leen casi cinco libros al año, 144 lo hacen en formato impreso y 43 en digital o electrónico. La preferencia por la lectura en formato impreso por estudiantes de CBI es apoyada por López (2016), que halló la misma preferencia mayoritaria (56\%) sobre la lectura digital (38 \%) y muy poco $(6 \%)$ en ambos formatos. Salado, Ramírez y Ochoa (2017) encontraron, respecto de la autopercepción de estudiantes de ciencias económico administrativas (CEA) sobre sus hábitos lectores en México, Argentina y España, que una cuarta parte de los universitarios tienen un nivel bajo y casi tres cuartas partes un nivel medio.

Los estudiantes en diversas disciplinas científicas manifiestan desiguales usos de internet, además de prácticas lectoras contrastantes en formatos digitales e impresos, con hábitos bajos y medios de lectura en estudiantes de CEA, sin encontrar diferencias de género en instituciones públicas (Salado, Ramírez y Ochoa, 2017). Por su parte, los estudiantes de CBI y CSH, en un muy alto porcentaje, emplean la computadora una sexta parte del día, y no se han encontrado diferencias significativas entre tales áreas disciplinares ni tampoco entre hombres y mujeres (Ramírez, 2006). La preferencia por el formato impreso se da tanto en estudiantes de CSH como de CBI (López, 2016) y los alumnos de CSH son los lectores más frecuentes (Salvador Oliván y Agustín Lacruz, 2015). De esta forma, se puede enunciar la siguiente proposición por demostrar: 
Hipótesis

Las interrogaciones de investigación se encaminaron a identificar los aspectos que inciden en la dedicación a la lectura por parte de los estudiantes universitarios. Se inquirió sobre la afectación del sector educativo, de las diversas disciplinas científicas y del género en el empleo de internet y de la práctica lectora de estudiantes de nivel superior. La hipótesis con nomenclatura de las variables fue la siguiente:

El género $(\mathrm{G})$, femenino $(\mathrm{F})$ o masculino $(\mathrm{M})$; las disciplinas, ciencias sociales y humanidades (CSH), ciencias básicas e ingeniería (CBI) y ciencias económico administrativas (CEA), y el sector educativo, privado $(\mathrm{Pv})$ o público $(\mathrm{Pb})$, inciden en el uso de internet (UIE), en la dedicación de lectura textual (DLT) y en la dedicación de lectura digital (DLD) de los alumnos universitarios.

Hipótesis particulares

1. El uso de internet de estudiantes se correlaciona positivamente con la dedicación a la lectura en texto impreso y con la lectura digital.

2. El género, las disciplinas y el sector educativo inciden favorablemente en el uso de internet de los alumnos universitarios.

3. El género, las disciplinas y el sector educativo inciden favorablemente en la dedicación a la lectura en texto impreso de los alumnos universitarios.

4. El género, las disciplinas y el sector educativo inciden favorablemente en la dedicación de lectura digital de los alumnos universitarios.

\section{Metodología}

\section{Enfoque y procedimiento de estudio}

La investigación tuvo un enfoque cuantitativo: se realizó la recolección de datos y un análisis estadístico para establecer patrones de comportamiento sobre los cuestionamientos del presente estudio, identificar la implicación del sector educativo, de las diversas disciplinas científicas y del género en el empleo de internet y de la práctica lectora de estudiantes de nivel superior, con el fin de comprobar las hipótesis establecidas. El alcance fue descriptivo-correlacional, pues se identificaron las características del fenómeno analizado (Hernández, Fernández y Baptista, 2014); en este caso, las características de las prácticas lectoras y del uso de internet de los estudiantes universitarios. 
Asimismo, el estudio fue explicativo, por indagar si el género, el tipo de institución y la disciplina incidieron en el uso de internet y en las prácticas lectoras de los estudiantes.

El procedimiento consistió en establecer tres variables independientes: género ( $\mathrm{F}$ y $\mathrm{M}$ ), disciplinas (CSH, CBI y CEA) y el sector educativo ( $\mathrm{PV}$ $\mathrm{o} \mathrm{Pb}$ ), y tres variables dependientes: uso de internet de estudiantes (UIE), dedicación a la lectura en texto impreso (DLT) y dedicación a la lectura digital (DLD). Los datos fueron obtenidos mediante una encuesta, codificados y capturados en el software estadístico SPSS 22. Se ejecutó una estadística descriptiva de las variables dependientes e independientes, después se realizó un análisis correlacional entre las tres variables dependientes y, finalmente, un análisis multivariado, donde se demuestra la influencia de las tres variables independientes, su efecto simple y combinado, sobre cada una de las tres variables dependientes. Se realizó un análisis de dependencia mediante un ANOVA multifactorial, en este caso, tres factores: género, disciplinas y sector educativo. Se obtuvieron así tres modelos lineales generales respecto a las tres variables a explicar: UIE, DLT y DLD.

\section{Instrumento}

El instrumento aplicado incluyó cuestionamientos sobre las horas semanales dedicadas al uso de internet, a la lectura digital, a lectura en formato impreso, el tipo de sector educativo, la disciplina y el género en una escala de frecuencia de horas semanales de 1, 2, 3, 4, 5 y más de 5 .

\section{Participantes}

La población se integró por 5455 estudiantes universitarios mexicanos, con una edad promedio de 24.18 años, con una desviación estándar de 5.21, distribuidos como se muestra en la Tabla 1.

\begin{tabular}{|l|c|c|}
\hline \multicolumn{1}{|c|}{ Factor } & N & Porcentaje \\
\hline Pública & 4622 & $85 \%$ \\
\hline Privada & 833 & $15 \%$ \\
\hline CBI & 1504 & $28 \%$ \\
\hline CEA & 985 & $18 \%$ \\
\hline CSH & 2966 & $54 \%$ \\
\hline Femenino & 3148 & $58 \%$ \\
\hline Masculino & 2307 & $42 \%$ \\
\hline
\end{tabular}

Tabla 1. Distribución de los participantes del estudio 


\section{Presentación y ANÁlisis de RESUltados}

\section{El uso de internet y dedicación lectora de estudiantes}

Los estudiantes universitarios presentaron, de acuerdo a la media obtenida, una mayor dedicación de horas al uso de internet seguida de la lectura en texto digital y en menor medida la dedicación a la lectura en texto impreso (Tabla 2).

\begin{tabular}{|l|c|c|c|}
\hline & Media & $\begin{array}{c}\text { Desviación } \\
\text { estándar }\end{array}$ & N \\
\hline Dedicación en texto impreso & 2.60 & 1.595 & 5455 \\
\hline Dedicación en texto digital & 2.81 & 1.680 & 5455 \\
\hline Dedicación a internet & 4.26 & 1.762 & 5455 \\
\hline
\end{tabular}

Tabla 2. Dedicación lectora y a uso de internet en horas semanales

\section{Dedicación lectora estudiantil de acuerdo a disciplina, género y tipo de institución}

La media en horas a la semana de dedicación a internet de la población fue ligeramente mayor en el género masculino. La media del grupo femenino de CSH de universidades públicas fue la menor entre todos los grupos y la del grupo masculino de CBI de universidades privadas fue la mayor entre todos los subgrupos. La media de los alumnos de CSH de universidades públicas fue la menor, seguida por los de CBI y la más alta fue la de CEA (Tabla 3).

\begin{tabular}{|l|l|c|c|c|c|c|c|}
\hline \multirow{2}{*}{ Disciplina } & \multirow{2}{*}{ Género } & Pública & & Privada & & Total & \\
\cline { 3 - 8 } & & Media & N & Media & N & Media & N \\
\hline \multirow{2}{*}{ CBI } & Femenino & 4.04 & 511 & 4.78 & 32 & 4.08 & 543 \\
\cline { 2 - 8 } & Masculino & 4.40 & 877 & 4.94 & 84 & 4.45 & 961 \\
\cline { 2 - 8 } & Total & 4.27 & 1388 & 4.90 & 116 & 4.32 & 1504 \\
\hline \multirow{2}{*}{ CEA } & Femenino & 4.15 & 514 & 4.43 & 104 & 4.20 & 618 \\
\cline { 2 - 8 } & Masculino & 4.54 & 306 & 4.87 & 61 & 4.59 & 367 \\
\cline { 2 - 8 } & Total & 4.30 & 820 & 4.59 & 165 & 4.35 & 985 \\
\hline
\end{tabular}




\begin{tabular}{|l|l|c|c|c|c|c|c|}
\hline \multirow{3}{*}{ CSH } & Femenino & 4.02 & 1607 & 4.54 & 380 & 4.12 & 1987 \\
\cline { 2 - 8 } & Masculino & 4.32 & 807 & 4.62 & 172 & 4.38 & 979 \\
\cline { 2 - 8 } & Total & 4.12 & 2414 & 4.57 & 552 & 4.20 & 2966 \\
\hline \multirow{4}{*}{ Total } & Femenino & 4.05 & 2632 & 4.53 & 516 & 4.13 & 3148 \\
\cline { 2 - 8 } & Masculino & 4.39 & 1990 & 4.75 & 317 & 4.44 & 2307 \\
\cline { 2 - 8 } & Total & 4.20 & 4622 & 4.62 & 833 & 4.26 & 5455 \\
\hline
\end{tabular}

Tabla 3. Dedicación lectora en internet por disciplina, género y tipo de institución

\section{Correlación entre dedicación lectora e internet de estudiantes}

Hipótesis 1. La dedicación lectora en texto impreso correlacionó positivamente con la de texto digital y tuvo una menor correlación positiva con la dedicación a internet, misma que correlacionó positivamente con la dedicación a la lectura en texto digital (Tabla 4).

\begin{tabular}{|l|c|c|c|}
\hline & $\begin{array}{c}\text { Dedicación lectora } \\
\text { en texto impreso }\end{array}$ & $\begin{array}{c}\text { Dedicación } \\
\text { lectora en texto } \\
\text { digital }\end{array}$ & $\begin{array}{c}\text { Dedicación } \\
\text { a internet }\end{array}$ \\
\hline $\begin{array}{l}\text { Dedicación lectora } \\
\text { en texto impreso }\end{array}$ & 1 & $.471^{\star \star}$ & $.267^{\star \star}$ \\
\hline $\begin{array}{l}\text { Dedicación lectora } \\
\text { en texto digital }\end{array}$ & & 1 & $.424^{\star \star}$ \\
\hline Dedicación a internet & & & 1 \\
\hline
\end{tabular}

** La correlación es significativa en el nivel 0,01 ( 2 colas)

Tabla 4. Correlaciones entre la dedicación a la lectura en texto impreso, digital e internet

\section{La incidencia de género, tipo de institución y disciplina en la dedicación a internet}

Hipótesis 2. El género, el tipo de institución y el área de estudios tienen un efecto simple y combinado en la dedicación a internet del estudiante universitario. La prueba de Levene realizada a los sujetos de estudio indicó un P-valor menor a 0.05 (Tabla 5), rechazando la hipótesis nula, que la varianza de error de la dedicación a internet es igual entre los subgrupos: género, tipo de institución, disciplina científica y sus combinaciones. Esta prueba sugirió proceder con el análisis lineal general.

\begin{tabular}{|c|c|c|c|}
\hline $\mathbf{F}$ & df1 & df2 & Sig. \\
\hline 4.458 & 11 & 5443 & .000 \\
\hline
\end{tabular}

Tabla 5. Prueba de Levene. Variable dependiente: dedicación a internet 
La prueba univariada general arrojó un modelo corregido de las horas de dedicación, el cual fue explicado por dos variables independientes, tipo de institución y género, que tuvieron valores cercanos a cero. Así se demostró su incidencia en las horas de dedicación a internet; contrariamente, la disciplina no tuvo incidencia en las horas dedicadas a internet (Tabla 6). Así, la hipótesis 2 fue soportada parcialmente, pues solamente el género y el tipo de institución incidieron en la dedicación a internet de los estudiantes universitarios.

\begin{tabular}{|l|c|c|c|c|c|}
\hline \multicolumn{1}{|c|}{ Origen } & $\begin{array}{c}\text { Tipo III de suma } \\
\text { de cuadrados }\end{array}$ & GI & $\begin{array}{c}\text { Cuadrático } \\
\text { promedio }\end{array}$ & F & Sig. \\
\hline Modelo corregido & $298.257^{\mathrm{a}}$ & 11 & 27.114 & 8.874 & .000 \\
\hline Interceptación & 32802.697 & 1 & 32802.697 & 10736.260 & .000 \\
\hline Privada & 83.931 & 1 & 83.931 & 27.470 & .000 \\
\hline Disciplina & 11.097 & 2 & 5.548 & 1.816 & .163 \\
\hline Sexo & 34.374 & 1 & 34.374 & 11.251 & .001 \\
\hline Privada * Disciplina & 5.956 & 2 & 2.978 & .975 & .377 \\
\hline Privada * Sexo & 1.700 & 1 & 1.700 & .556 & .456 \\
\hline Disciplina * Sexo & 4.580 & 2 & 2.290 & .749 & .473 \\
\hline $\begin{array}{l}\text { Privada * Disciplina } \\
\text { * Sexo }\end{array}$ & 1.902 & 2 & .951 & .311 & .733 \\
\hline Error & 16630.100 & 5443 & 3.055 & & \\
\hline Total & 115938.000 & 5455 & & & \\
\hline Total corregido & 16928.357 & 5454 & & & \\
\hline
\end{tabular}

a. $\mathrm{R}$ al cuadrado $=.018$ ( $\mathrm{R}$ al cuadrado ajustada $=.016)$

Tabla 6. Prueba de efectos inter-sujetos. Variable dependiente: dedicación semanal a internet

\section{Incidencia de género, tipo de institución y disciplina en la dedicación a la lectura en texto impreso}

La media poblacional total de la dedicación a la lectura en texto impreso fue de 2.60. La media de la dedicación a la lectura en texto impreso del género femenino fue mayor al masculino y este tuvo una media menor a la de toda la población. La media del grupo femenino de CEA de universidades públicas fue la menor entre todos los grupos, mientras que la media del grupo masculino de CSH de universidades públicas fue la mayor (Tabla 7).

\begin{tabular}{|l|l|c|c|c|c|c|c|}
\hline \multirow{2}{*}{ Disciplina } & \multirow{2}{*}{ Género } & \multicolumn{2}{|c|}{ Pública } & \multicolumn{2}{c|}{ Privada } & \multicolumn{2}{c|}{ Total } \\
\cline { 3 - 8 } & & Media & $\mathbf{N}$ & Media & N & Media & N \\
\hline \multirow{2}{*}{$\mathrm{CBI}$} & Femenino & 2.42 & 511 & 2.31 & 32 & 2.41 & 543 \\
\cline { 2 - 9 } & Masculino & 2.35 & 877 & 2.37 & 84 & 2.35 & 961 \\
\cline { 2 - 8 } & Total & 2.37 & 1388 & 2.35 & 116 & 2.37 & 1504 \\
\hline
\end{tabular}




\begin{tabular}{|l|l|c|c|c|c|c|c|}
\hline \multirow{4}{*}{ CEA } & Femenino & 2.29 & 514 & 2.59 & 104 & 2.34 & 618 \\
\cline { 2 - 8 } & Masculino & 2.30 & 306 & 2.44 & 61 & 2.32 & 367 \\
\cline { 2 - 8 } & Total & 2.30 & 820 & 2.53 & 165 & 2.34 & 985 \\
\hline \multirow{3}{*}{ CSH } & Femenino & 2.77 & 1607 & 2.72 & 380 & 2.76 & 1987 \\
\cline { 2 - 8 } & Masculino & 2.90 & 807 & 2.69 & 172 & 2.87 & 979 \\
\cline { 2 - 8 } & Total & 2.82 & 2414 & 2.71 & 552 & 2.80 & 2966 \\
\hline \multirow{3}{*}{ Total } & Femenino & 2.61 & 2632 & 2.67 & 516 & 2.62 & 3148 \\
\cline { 2 - 8 } & Masculino & 2.57 & 1990 & 2.56 & 317 & 2.56 & 2307 \\
\cline { 2 - 8 } & Total & 2.59 & 4622 & 2.63 & 833 & 2.56 & 5455 \\
\hline
\end{tabular}

Tabla 7. Dedicación a la lectura en texto impreso

Hipótesis 3. El género, el tipo de institución y el área de estudios tienen un efecto simple y combinado en la dedicación del estudiante universitario a la lectura en texto impreso. La prueba de Levene realizada a los sujetos de estudio indicó que la media de los diferentes subgrupos es diferente (Tabla 8). La prueba univariada general produjo un modelo corregido sobre las horas de dedicación a la lectura en texto impreso, explicado por una de las variables independientes. Se muestra que el tipo de institución y el género no tuvieron valores cercanos a cero, sin efecto alguno, no tuvieron significancia sobre la dedicación a la lectura en texto impreso. Contrariamente, la disciplina tuvo un valor igual a cero, por tanto incidió en la dedicación en horas de lectura de textos impresos (Tabla 9). Con esta prueba se afirma que la hipótesis 3 fue soportada parcialmente, pues el género y el tipo de institución no incidieron, sólo la disciplina científica tuvo efecto en la dedicación a la lectura de textos impresos de los estudiantes universitarios.

\begin{tabular}{|c|c|c|c|}
\hline $\mathbf{F}$ & df1 & df2 & Sig. \\
\hline 6.610 & 11 & 5443 & .000 \\
\hline
\end{tabular}

Tabla 8. Prueba de Levene. Variable dependiente: dedicación lectora en texto impreso

\begin{tabular}{|l|c|c|c|c|c|}
\hline \multicolumn{1}{|c|}{ Origen } & $\begin{array}{c}\text { Tipo III de suma } \\
\text { de cuadrados }\end{array}$ & gl & $\begin{array}{c}\text { Cuadrático } \\
\text { promedio }\end{array}$ & $\mathrm{F}$ & Sig. \\
\hline Modelo corregido & $287.961^{\mathrm{a}}$ & 11 & 26.178 & 10.484 & .000 \\
\hline Interceptación & 10362.302 & 1 & 10362.302 & 4149.902 & .000 \\
\hline Privada & .098 & 1 & .098 & .039 & .843 \\
\hline Disciplina & 82.476 & 2 & 41.238 & 16.515 & .000 \\
\hline Sexo & .030 & 1 & .030 & .012 & .913 \\
\hline Privada * Disciplina & 11.666 & 2 & 5.833 & 2.336 & .097 \\
\hline
\end{tabular}




\begin{tabular}{|l|c|c|c|c|c|}
\hline $\begin{array}{l}\text { Privada } \\
{ }^{*} \text { Sexo }\end{array}$ & .399 & 1 & .399 & .160 & .689 \\
\hline Disciplina * Sexo & 1.418 & 2 & .709 & .284 & .753 \\
\hline $\begin{array}{l}\text { Privada } \\
{ }^{*} \text { Disciplina } \\
\text { * Sexo }\end{array}$ & 1.548 & 2 & .774 & .310 & .733 \\
\hline Error & 13591.165 & 5443 & 2.497 & & \\
\hline Total & 50651.000 & 5455 & & & \\
\hline Total corregido & 13879.127 & 5454 & & & \\
\hline
\end{tabular}

a. $\mathrm{R}$ al cuadrado $=.021$ ( $\mathrm{R}$ al cuadrado ajustada $=.019)$

Tabla 9. Efectos inter-sujetos. Variable dependiente: dedicación lectora en texto impreso

\section{La incidencia del género, el tipo de institución y el área disciplinar en la dedicación a la lectura digital}

La media en la dedicación a la lectura digital de los estudiantes de CBI fue la mayor entre los alumnos de todas las disciplinas, los de CSH le siguieron y los de CEA fueron quienes registraron el menor valor promedio. Adicionalmente, la media de la dedicación a la lectura digital del género masculino fue mayor al femenino. La media más alta en la dedicación en la lectura digital se presentó en el grupo masculino de CBI de universidades privadas, mientras que la más baja fue la del grupo femenino del área de CEA de universidades públicas (Tabla 10).

\begin{tabular}{|l|l|c|c|c|c|c|c|}
\hline \multirow{2}{*}{ Disciplina } & \multirow{2}{*}{ Género } & Pública & & Privada & & Total & \\
\cline { 3 - 8 } & & Media & $\mathbf{N}$ & Media & N & Media & N \\
\hline \multirow{3}{*}{ CBI } & Femenino & 2.65 & 511 & 3.66 & 32 & 2.71 & 543 \\
\cline { 2 - 8 } & Masculino & 2.91 & 877 & 3.64 & 84 & 2.97 & 961 \\
\cline { 2 - 8 } & Total & 2.81 & 1388 & 3.65 & 116 & 2.88 & 1504 \\
\hline \multirow{3}{*}{ CEA } & Femenino & 2.52 & 514 & 2.77 & 104 & 2.56 & 618 \\
\cline { 2 - 8 } & Masculino & 2.77 & 306 & 3.43 & 61 & 2.88 & 367 \\
\cline { 2 - 8 } & Total & 2.62 & 820 & 3.01 & 165 & 2.68 & 985 \\
\hline \multirow{2}{*}{ CSH } & Femenino & 2.69 & 1607 & 3.07 & 380 & 2.76 & 1987 \\
\cline { 2 - 8 } & Masculino & 2.95 & 807 & 2.97 & 172 & 2.96 & 979 \\
\cline { 2 - 8 } & Total & 2.78 & 2414 & 3.04 & 552 & 2.83 & 2966 \\
\hline
\end{tabular}




\begin{tabular}{|l|l|l|l|l|l|l|l|}
\hline \multirow{3}{*}{ Total } & Femenino & 2.65 & 2632 & 3.05 & 516 & 2.71 & 3148 \\
\cline { 2 - 8 } & Masculino & 2.91 & 1990 & 3.24 & 317 & 2.95 & 2307 \\
\cline { 2 - 8 } & Total & 2.76 & 4622 & 3.12 & 833 & 2.81 & 5455 \\
\hline
\end{tabular}

Tabla 10. Estadísticos descriptivos. Lectura digital

Hipótesis 4. El género, el tipo de institución y el área de estudios tienen un efecto simple y combinado en la dedicación del estudiante universitario a la lectura digital. La prueba de Levene realizada a los sujetos de estudio indicó, respecto a dedicación a la lectura digital, que la media de los subgrupos analizados fue diferente (Tabla 11).

\begin{tabular}{|c|c|c|c|}
\hline F & df1 & df2 & Sig. \\
\hline 2.396 & 11 & 5443 & .006 \\
\hline
\end{tabular}

Tabla 11. Prueba de Levene. Variable dependiente: lectura digital

La prueba univariada general arrojó un modelo corregido sobre las horas de dedicación a la lectura digital, el cual fue explicado por las tres variables independientes: tipo de institución, la disciplina y el género, debido a que tuvieron valores cercanos a cero, igual que todas sus combinaciones. Con esta prueba se puede afirmar que la hipótesis 4 fue soportada totalmente, ya que el género, la disciplina y el tipo de institución incidieron, al ser significativas, en la dedicación a la lectura digital de los estudiantes universitarios.

\begin{tabular}{|l|c|c|c|c|c|}
\hline \multicolumn{1}{|c|}{ Origen } & $\begin{array}{c}\text { Tipo III de suma } \\
\text { de cuadrados }\end{array}$ & gl & $\begin{array}{c}\text { Cuadrático } \\
\text { promedio }\end{array}$ & F & Sig. \\
\hline Modelo corregido & $240.532^{\mathrm{a}}$ & 11 & 21.86 & 7.859 & .000 \\
\hline Interceptación & 14792.244 & 1 & 14792.24 & 5316.189 & .000 \\
\hline Privada & 105.551 & 1 & 105.55 & 37.934 & .000 \\
\hline Disciplina & 28.421 & 2 & 14.21 & 5.107 & .006 \\
\hline Sexo & 19.735 & 1 & 19.73 & 7.093 & .008 \\
\hline Privada * Disciplina & 33.381 & 2 & 16.69 & 5.998 & .002 \\
\hline Privada * Sexo & .626 & 1 & .626 & .225 & .635 \\
\hline Disciplina * Sexo & 13.448 & 2 & 6.724 & 2.417 & .089 \\
\hline Privada*Disciplina* Sexo & 14.730 & 2 & 7.365 & 2.647 & .071 \\
\hline Error & 15145.095 & 5443 & 2.782 & & \\
\hline Total & 58602.000 & 5455 & & & \\
\hline Total corregido & 15385.627 & 5454 & & & \\
\hline
\end{tabular}

Tabla 12. Efectos inter-sujetos. Variable dependiente: dedicación lectora digital 


\section{Discusión}

La investigación confirmó correlaciones positivas entre el uso de internet y la lectura digital, pero mayor con la lectura en formato impreso de los estudiantes universitarios. Lo anterior es parecido a los resultados de Salvador Oliván y Agustín Lacruz (2015) en cuanto a que la lectura en formato impreso es preferida sobre la digital.

Los estudiantes masculinos universitarios dedicaron mayor número de horas a internet, lo que coincide con lo argumentado por Moya y Gerber (2016), Salado, Ramírez y Ochoa (2017). Además, apoya la idea de Curbelo y Moreira (2014) sobre que los hombres tienen mayor dominio en las TIC.

Respecto al tipo de educación, se encontró que los estudiantes de universidades privadas tienen más horas de dedicación al uso de internet. Además, los estudiantes de CEA dedican mayor número de horas a internet, confirmando los resultados de la investigación de Salado, Ramírez y Ochoa (2017), acerca de que los alumnos de CEA tienen un alto índice de afinidad tecnológica y un elevado índice de lectura por internet.

En lo referente a la lectura impresa, los hombres tienen mayor dedicación en comparación con las mujeres, lo cual coincide con Salado, Ramírez y Ochoa (2017). Por otra parte, los alumnos de universidades públicas dedican mayor número de horas a lectura impresa; lo anterior difiere de los resultados de Amiama-Espaillat y Mayor-Ruiz (2017). Los alumnos de CSH presentaron mayor dedicación a la lectura impresa, lo que coincide con lo afirmado por Salvador Oliván y Agustín Lacruz (2015).

Otra controversia se dio en la lectura digital, a la cual los hombres presentaron mayor dedicación, apoyando los resultados de Curbelo y Moreira (2014) y refutando la igualdad de géneros en la dedicación de lectura mostrada por Salado, Ramírez, y Ochoa (2017).

Respecto al tipo de universidad, se halló que en las universidades públicas se lee menos en formato digital que en las instituciones privadas, convergiendo con Arias (2015). Además, los resultados apoyaron lo expuesto por Amiama-Espaillat y Mayor-Ruiz (2017), respecto de que la competencia lectora digital es mayor en universitarios de educación privada. Por último, las áreas científicas de los estudiantes, los de CBI presentaron mayor dedicación a la lectura digital, contrario a lo argumentado por Ramírez (2006) y Cornejo et al. (2012) sobre la preferencia de los universitarios del área de CBI a la lectura en formato impreso. 


\section{CONCLUSIONES Y CONSIDERACIONES FINALES}

Se determinó que la dedicación a internet presenta una asociación positiva con la dedicación a la lectura impresa y a la lectura digital. Además, los hombres se dedican más que las mujeres a la lectura. Cabe destacar también la dedicación a la lectura impresa por ambos sexos de los estudiantes de CSH y por los masculinos de CBI del ámbito público.

En el sentido del uso de internet de los universitarios, se sugiere facilitar la promoción y dedicación a internet tanto como la dedicación a la lectura. No se debe prejuzgar que la dedicación a internet afecta la lectura pues se demostró que la dedicación a la lectura está asociada efectivamente con el uso de internet. Por ello, las bibliotecas universitarias deben ser espacios idóneos y propicios, además de la perspectiva convencional para la lectura en formato impreso, para la lectura en formato digital.

Con relación a la educación universitaria privada versus pública, las bibliotecas universitarias públicas deben evolucionar de espacios para textos impresos a textos digitales, incluso documentos y materiales digitalizados precargados por asignaturas, pantallas visuales ad hoc para el análisis, organización, comparación, identificación de ideas y autores, entre otras operaciones cognitivas que potencialicen este tipo de formatos. Incluso se podrían estimular las emociones a partir de las prácticas lectoras de textos digitales, por ejemplo, mediante tecnologías de pantallas táctiles. Además, diseñar y ampliar bibliotecas digitales, con bibliografía propia diseñada para la lectura en formato digital, como imágenes digitales de textos antiguos originales de ciencias sociales, humanidades, básicas e ingeniería como estimulantes afectivos de las prácticas lectoras.

Debido a que en la universidad privada se tiene una mayor práctica de la lectura digital, esto debe encaminar políticas educativas gubernamentales para lograr un mayor equilibrio en las universidades públicas. Esto es, que en las bibliotecas universitarias públicas también se fomente la lectura digital, se adapten materiales e infraestructura para conexiones y uso de internet. Así, se optimizan los recursos bibliográficos al compartirlos mediante internet en formato digital.

Con relación al género y las diversas disciplinas científicas, destaca que los hombres de CBI de universidades públicas se dedican más a la lectura en texto impreso, esto confirma que el género masculino tiene mayor dedicación. Esto llevaría a cuestionar los aspectos culturales o económicos familiares que no favorecen las prácticas lectoras del género femenino.

Finalmente, se puede determinar que las CSH favorecen la práctica de la lectura, quizás por ser una actividad disciplinar muy arraigada. En este 
sentido, las otras disciplinas podrían equilibrar su diseño curricular e incentivar actividades lectoras relacionadas con las CSH para que se complementen y estimulen competencias lectoras en los estudiantes universitarios de las demás disciplinas. En este sentido, las bibliotecas de las universidades deben ser espacios propios para la lectura en formato digital y contener textos de las CSH además de las propias de CBI y de CEA.

\section{REFERENCIAS}

Álvarez, Didier, Yicel Giraldo, Norfi Ocampo, Luz Guerra, Liliana Melgar y Maricela Gómez. 2009. "Representaciones bibliotecarias sobre la biblioteca pública, la lectura, el lector, la promoción y la animación a la lectura en Medellin, Colombia". Investigación Bibliotecológica: archivonomía, bibliotecología e información 23 (47): 161-188. http://dx.doi.org/10.22201/iibi.0187358xp.2009.49.21395

Amiama-Espaillat, Cristina y Cristina Mayor-Ruiz. 2017. "Lectura digital en la competencia lectora: La influencia en la Generación Z de la República Dominicana”. Comunicar 25 (52): 105-114. https://doi.org/10.3916/C52-2017-10

Arias, Walter. 2015. "Tecnologías de la información y la comunicación en colegios públicos y privados de Arequipa". Interacciones 1 (1): 11-28. http://dx.doi. org/10.24016/2015.v1n1.1

Conaculta (Consejo Nacional para la Cultura y las Artes). 2015. Encuesta Nacional de Lectura y Escritura 2015. México: Dirección General de Publicaciones.

Cornejo, Jorge, María Roble, Carmen Barrero y Ana Martín. 2012. "Hábitos de lectura en alumnos universitarios de carreras de ciencia y de tecnología". Revista Eureka sobre Enseñanza y Divulgación de las Ciencias 9 (1): 155-163. http://www. redalyc.org/articulo.oa?id=92024530011

Curbelo, Dayana y Natalia Moreira. 2014. "Una mirada de género sobre el uso de las tecnologías por parte de los adolescentes". Trabajo presentado en las XIII Jornadas de Investigación de la Facultad de Ciencias Sociales. Montevideo, 14-16 de septiembre.

García, Belén y Gala Arias. 2014. "Papel vs. digital: hábitos de lectura de los estudiantes de la UEM". Caracteres. Estudios culturales y críticos de la esfera digital 3 (1): 250-271. http://revistacaracteres.net/wpcontent/uploads/2014/05/Caracteresvol3n1mayo2014-papel-digital-lectura-estudiantes.pdf

Hernández, Roberto, Carlos Fernández y Lucio Baptista. 2014. Metodología de la Investigación. México: McGraw Hill.

López, Karen. 2016. "Prácticas de lectura digital de estudiantes universitarios." Enseñanza \& teaching 34 (1) (2016):57-92. http://dx.doi.org/10.14201/et20163415792

Marchesi, Álvaro. 2001. "Lectura y bibliotecas escolares: un objetivo estratégico para las políticas educativas y culturales", en Cultura escrita. Lectura y bibliotecas escolares, Inés Miret y Cristina Armendano, 7-9. Madrid: Organización de Estados Iberoamericanos OEI.

Moya, Cristóbal y Mónica Gerber. 2016. "La lectura en formatos digitales en el Chile actual: nuevas prácticas y viejas desigualdades". Revista Chilena de Literatura (94): 5977. https://revistaliteratura.uchile.cl/index.php/RCL/article/view/44970/47052 
Ramírez, Elsa. 2006. "The impact of the internet on the reading and information practices of a university student community: the case of UNAM". New Review of Libraries and Lifelong Learning 4 (1): 137-157. https://doi.org/10.1080/1468994042000240287

Ramírez, Elsa. 2010. “¿Qué leer? ¿Qué es la lectura?”. Investigación Bibliotecológica: archivonomía, bibliotecología e información 23 (47): 161-188. http://dx.doi. org/10.22201/iibi.0187358xp.2009.47.16961

Ramírez, Elsa. 2016. "De la promoción de la lectura por placer a la formación integral de lectores”. Investigación Bibliotecológica: Archivonomía, Bibliotecología e Información 30 (69): 95-120. http://dx.doi.org/10.1016/j.ibbai.2016.04.014

Salado, Lilian, Alberto Ramírez y Reyna Ochoa. 2017. "Hábitos de lectura y afinidad tecnológica de los estudiantes universitarios: estudio comparativo de cinco universidades de habla hispana”. Estudios Lambda. Teoría y práctica de la didáctica en lengua y literatura (2): 1-24. http://estudioslambda.uson.mx/revistas/articulos/19-15-16-2-r2_art01.pdf

Salvador Oliván, José Antonio y Ma. del Carmen Agustín Lacruz. 2015. "Hábitos de lectura y consumo de información en estudiantes de la Facultad de Filosofía y Letras de la Universidad de Zaragoza”. Anales de Documentación 18 (1): 1-15. http://dx.doi.org/10.6018/analesdoc.18.1.201971

Shandana, Arshad y Ahmed Hafsa. 2015. "Hindrances in the usage of information and communication technology among public and private sector universities". Scholedge International Journal of Multidisciplinary \& Allied Studies 2 (5): 55-67. http://thescholedge.org/index.php/sijmas/article/view/146/118

Torres, Carlos y Ana Arras. 2011. "Percepción de estudiantes de educación superior sobre sus competencias en las TIC en las universidades Autónoma de Chihuahua y Veracruzana”. Apertura 3 (2): 1-29. http://www.redalyc.org/articulo.oa?id=68822737009

Zempoalteca, Beatriz, Jorge Barragán, Juan González y Teresa Guzmán. 2017. "Formación en TIC y competencia digital en la docencia en instituciones públicas de educación superior”. Apertura 9 (1): 80-96. http://dx.doi.org/10.18381/Ap.v9n1.922

Para citar este texto:

Robles-Francia, Víctor Hugo, Adriana Mariela de la Cruz Caballero, Aída Dinorah García Álvarez, Lenin Martínez Pérez y Jorge Rebollo Meza. 2019. "La educación privada y el área científica favorecen la lectura de universitarios". Investigación Bibliotecológica: archivonomia, bibliotecología e información 33 (78): 63-79.

http://dx.doi.org/10.22201/iibi.24488321xe.2019.78.57971 